\title{
Dosimetric comparison between XR-RV3 and EBT2 radiochromic film in megavoltage photon beams
}

\author{
I. Setilo* and F.C.P. du Plessis \\ Medical Physics Department, Faculty of Health Sciences, University of the Free State, Bloemfontein 9300, \\ South Africa
}

Technical note

\author{
*Corresponding author: \\ Dr. Itumeleng Setilo, \\ Fax: +27 514443822 \\ E-mail: itumelengs@gmail.com \\ Revised: March 2015 \\ Accepted: May 2015 \\ Int. J. Radiat. Res., April 2016; \\ 14(2): 149-152 \\ DOI: $10.18869 /$ acadpub.ijrr.14.2.81
}

\begin{abstract}
Background: Radiochromic film is used for radiation dose measurement, XRRV3 is used in fluoroscopy and EBT2 film in radiation therapy. The aim was to determine if the dosimetric properties of these two films are comparable in megavolt photon beams. Materials and Methods: Comparison measurements included: calibration curves, heterogeneous phantom dose profiles, and nasopharynx dose distribution measurement. Results: Both film types required 24 hours to stabilize. Their heterogeneous phantom dose profiles were similar, and their dose distributions for a nasopharynx treatment were within $3 \% / 3 \mathrm{~mm}$. Conclusion: Dose distributions between both films showed good correlation and XR-RV3 film can be used in radiotherapy for quality control checks.
\end{abstract}

Keywords: XR-RV3, EBT2, Calibration curves, heterogeneous.

\section{INTRODUCTION}

Quality control (QC) is critical for optimal and accurate radiation treatment of cancer patients. This entails measurement of sentinel parameters on all equipment used for imaging, tumor location, treatment planning, and setup correction and radiation delivery. Film dosimetry plays an integral part in QC procedures that verifies treatment planning and radiation dose delivery to the patient, especially during commissioning of this equipment. It also records dose patterns during QA tests of mechanical and isocentric alignment and stability of radiation machines as well as its output beam characteristics. Advantages of film include: high spatial resolution, reproducibility, dose integration, stability, and 2-D dose distribution measurement (1,2). Currently, Radiochromic film is a popular tool for dosimetry since it is not light sensitive, needs no processing, is stable, and can be used in water.

In Radiochromic film, ionizing radiation interacts with the active lithium salt of pentacosa-10,12-diynoic acid (LiPDCA) leading to the formation of free radicals that sets on polymerization $(3,4)$. This reaction requires at least 24 hours to reach stabilization (5). The Compton Effect dominates at radio therapeutic energies, and depends on the electronic density of the film. As a result its density should match that of normal tissue to reflect the patient dose accurately. EBT2 film has been designed with this goal in mind. The Photoelectric effect is dominant at diagnostic radiology energies and depends on the effective atomic number $\left(Z_{\text {eff }}\right)$ of the film. The polyester base of XR-RV3 film has higher $Z_{\text {eff }}$ and physical density compared to EBT2, to increase its sensitivity in the kilo-voltage (fluoroscopic) energy range (6). $\mathrm{XR}-\mathrm{RV} 3$ is used for fluoroscopy entrance dose measurement.

Existing literature does not compare these two films, but mostly focus on different generations of EBT film. Arjomandy et al. (8) showed that the EBT2 film has a small energy dependence for the different energies and modalities. Brown et al. (9) showed that dependency on energy of the EBT 
and EBT2 weakens when the film is exposed to larger doses, whereas the EBT3 shows weaker energy dependence. The aim of this study was to compare dosimetric properties of XR-RV3 and EBT2 film in megavoltage photon beams.

\section{MATERIALS AND METHODS}

EBT2 and XR-RV3 each have four layers shown in tables 1 and 2. EBT is semi-transparent while XR-RV3 film has a yellow polyester coating that faces the radiation source during dose measurement (6). An EPSON Perfection V330 Photo scanner (Scan software version 3.9.0.0US) was used to scan the film (7). The Colour depth was set to 16 bit depth for each of the RGB-channels and scan resolution of $72 \mathrm{dpi}$ in reflection mode which is acceptable for both film types $(9,10)$. After irradiation, the films were left for 24 hours to complete the polymerization process before they were scanned (8). Dreindl et al. (14) indicated that improvement in consistency of film dosimetry is achieved by following a fixed protocol. The red channel of the film images was used for analysis (11).

For calibration curve comparison, 5 film pieces $\left(2 \times 2.5 \mathrm{~cm}^{2}\right)$ were taken from each film type and each piece was sandwiched at a depth of dose maximum between layers of RW3 with total thickness of $10 \mathrm{~cm}$. The source-to-surface distance was $100 \mathrm{~cm}$ in a $10 \times 10 \mathrm{~cm}^{2}$ field. The pieces were irradiated to doses of $0,80,200$, 500 , and 550 monitor units (MU) respectively for $6 \mathrm{MV}$. After scanning, the average pixel value in a central region of interest $\left(1 \times 1 \mathrm{~cm}^{2}\right)$ was obtained the relative optical density was calculated with a method from Girard F et al. (13).

Film response for 6 and 15 MV photon energies were measured in a heterogeneous phantom as seen in figure 1. Each film strip was irradiated to $500 \mathrm{MU}$ at an SSD of $100 \mathrm{~cm}$ in a $5 \times 5 \mathrm{~cm}^{2}$ field. The field was bisected by slabs of $2 \mathrm{~cm}$ lead and $10 \mathrm{~cm}$ polystyrene and $5 \mathrm{~cm}$ thick RW3 was used for backscattering.

A radiation plan was set up for $6 \mathrm{MV}$ for a nasopharynx site in a Rando phantom. The radiation dose was delivered with the EBT2 film in the phantom and repeated for XR-RV3 film. The scanned film data were analysed with OmniPro I'MRT software version 1.7.

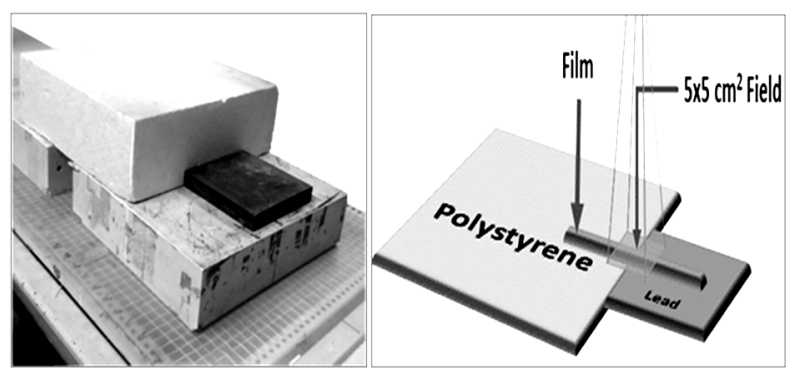

Figure 1. Heterogeneous phantom setup to test dosimetry properties in enhanced scatter and beam filtering conditions.

Table 1. Structure and composition of gafchromic EBT2 film.

\begin{tabular}{|c|c|c|c|c|c|c|c|}
\hline \multirow{2}{*}{ Layer } & \multirow{2}{*}{$\begin{array}{c}\text { Nominal } \\
\text { thickness }(\boldsymbol{\mu m})\end{array}$} & \multirow{2}{*}{$\begin{array}{c}\text { Density } \\
\left(\mathbf{g} / \mathbf{c m}^{\mathbf{2}}\right)\end{array}$} & $\mathbf{H}$ & $\mathbf{L i}$ & $\mathbf{C}$ & $\mathbf{0}$ & Al \\
\cline { 4 - 8 } & 50 & 1.35 & $36.4 \%$ & $0.0 \%$ & $45.5 \%$ & $18.2 \%$ & $0.0 \%$ \\
\hline Smooth polyester film base & 20 & 1.2 & $57.1 \%$ & $0.0 \%$ & $33.3 \%$ & $9.5 \%$ & $0.0 \%$ \\
\hline Acrylic adhesive & 28 & 1.2 & $56.8 \%$ & $0.6 \%$ & $27.6 \%$ & $13.3 \%$ & $1.6 \%$ \\
\hline Active layer (assumes 7.5\% moisture) & 175 & 1.35 & $36.4 \%$ & $0.0 \%$ & $45.5 \%$ & $18.2 \%$ & $0.0 \%$ \\
\hline Smooth polyester film base & & &
\end{tabular}

Table 2. Structure and composition of XR-RV3 film.

\begin{tabular}{|c|c|c|c|c|c|c|c|c|c|}
\hline \multirow{2}{*}{ Layer } & \multirow{2}{*}{$\begin{array}{c}\text { Nominal } \\
\text { thickness }(\boldsymbol{\mu m})\end{array}$} & $\begin{array}{l}\text { Density } \\
\left(\mathbf{g} / \mathbf{c m}^{2}\right)\end{array}$ & $\mathbf{H}$ & $\mathbf{L i}$ & $\mathbf{C}$ & $\mathbf{O}$ & $\mathbf{A l}$ & $\mathbf{C l}$ & $\mathbf{B r}$ \\
\hline Smooth polyester film base (Yellow) & 97 & 1.35 & $36.4 \%$ & $0.0 \%$ & $45.5 \%$ & $18.2 \%$ & $0.0 \%$ & & \\
\hline Acrylic adhesive & 20 & $\sim 1.2$ & $57.1 \%$ & $0.0 \%$ & $33.3 \%$ & $9.5 \%$ & $0.0 \%$ & & \\
\hline Active layer (assumes 7.5\% moisture) & 17 & $\sim 1.2$ & $56.8 \%$ & $0.6 \%$ & $27.6 \%$ & $13.3 \%$ & $1.6 \%$ & & \\
\hline Smooth polyester film base (White) & 97 & 1.35 & $42.3 \%$ & $0.0 \%$ & $33.8 \%$ & $21.6 \%$ & $0.0 \%$ & $1.2 \%$ & $1.2 \%$ \\
\hline
\end{tabular}




\section{RESULTS}

Figure 2 shows the time-evolution of the dose response curves for both film types. The calibration curves were measured at $2,10,24$ and 46 hours post irradiation and indicate that XR-RV3 is less sensitive than EBT2. Figure 3 shows dose profiles for a $6 \mathrm{MV}$ photon beam (left) and 15 MV photon beam (right) for a $5 \times 5$ $\mathrm{cm}^{2}$ field in the heterogeneous phantom. Both graphs indicate that XR-RV3 has similar sensitivity to EBT2. Figure 4 compared the dose distribution of each film type for a nasopharynx treatment. The XR-RV3 and EBT2 results are in good correlation with each other, which is obtainable after proper calibration of these films.

\section{DISCUSSION}

$\mathrm{XR}-\mathrm{RV} 3$ is less sensitive to photon radiation and post irradiation polymerization causes the same broadening in their respective ROD over 46 hours. Both films stabilized after 24 hours which agrees well with literature findings. Comparison between table 1 and 2 shows halogens $(\mathrm{Cl}$ and $\mathrm{Br})$ in the polyester layer of XR-RV3 but figure 3 showed that dose profiles from both films are nearly identical at megavoltage energies. McCabe et al. (6) showed that the white side of XR-RV3 absorbed more radiation compared to the orange side. The phantom (figure 1) was designed to cause differential filtering of the soft beam components but this did not cause a deviation in the dose profiles in figure 3. For challenging heterogeneous geometries such as head-andneck treatment, the example in figure 4 shows that the dose distribution obtained with both films are comparing within $2 \mathrm{~mm} / 2 \%$ in the nasopharynx radiation case for the majority of data points. Some discrepancies occur in the inferior part of the 250 cGy isodose line, but the dose gradient here are not steep. EBT2 film has been proven to be accurate at measuring dose distributions (12). Since XR-RV3 results compares well with EBT2 film in this study, it implies satisfactory accuracy as well.

\section{CONCLUSION}

XR-RV3 is more sensitive to radiation at megavolt photon energies when compared to EBT2. When properly calibrated, these films are equivalent at these energies despite their different atomic composition in the bottom polyester layer were XR-RV3 contains halogens. This is evident in the results shown in figure 3 and 4. It can be expected since the Compton Effect dominates at these energies. XR-RV3 film can be used for megavoltage photon beam dosimetry with the same degree of accuracy as EBT2 film.

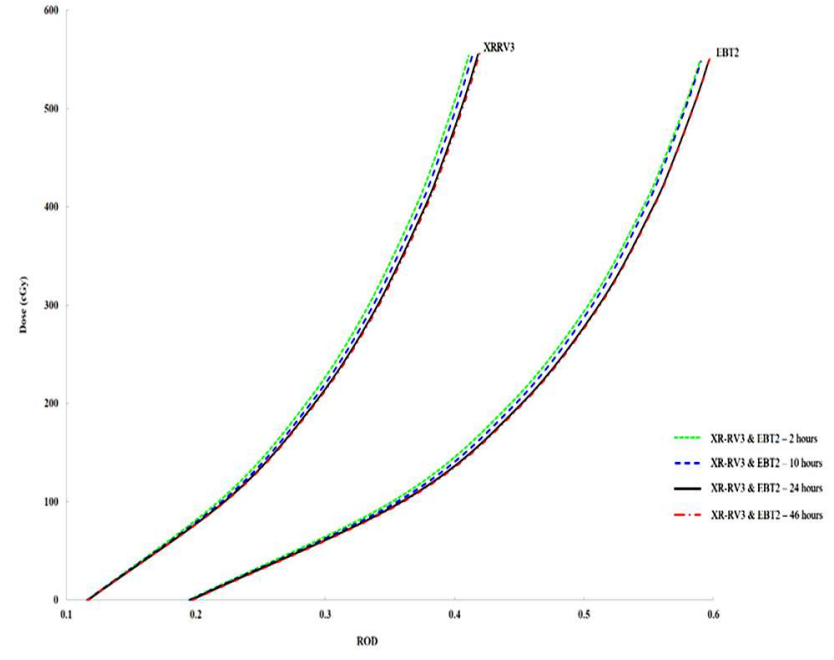

Figure 2. Calibration curves for EBT2 and XR-RV3 film between 2 and 46 hours.

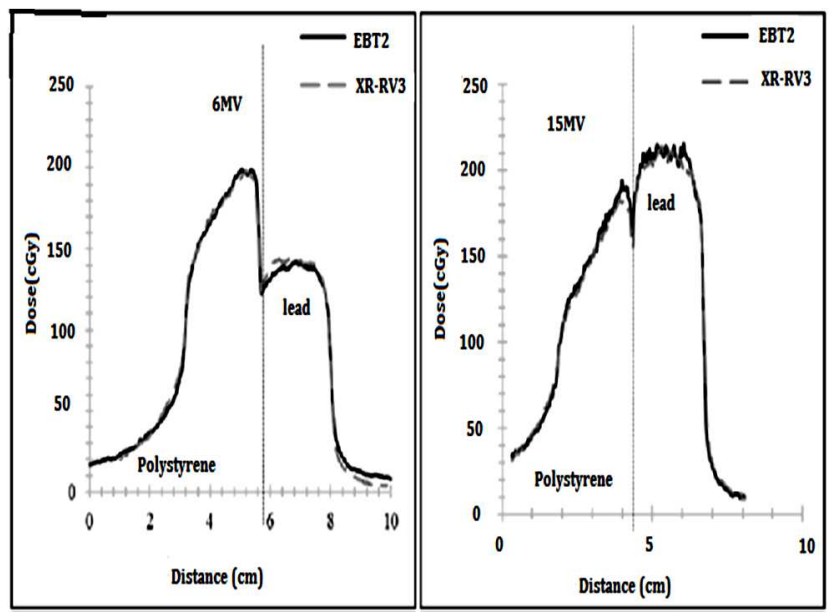

Figure 3. Dose profiles measured for the films in the heterogeneous phantom for $6 \mathrm{MV}$ and $15 \mathrm{MV}$ photon beams.

Int. J. Radiat. Res., Vol. 14 No. 2, April 2016 


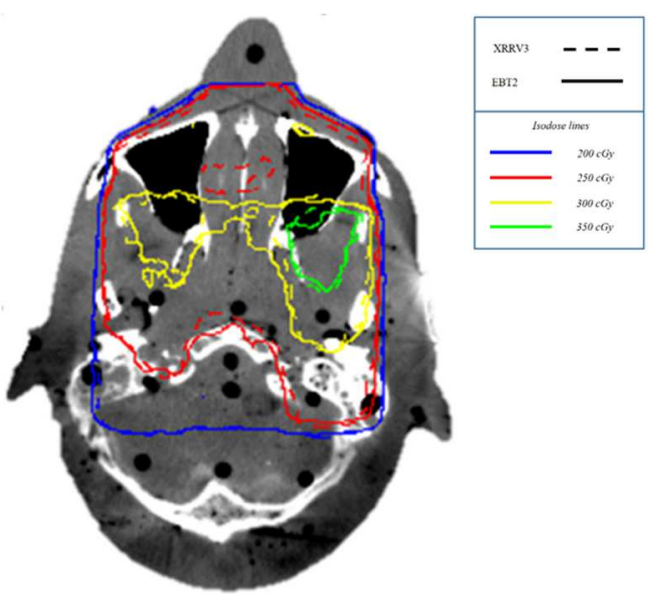

Figure 4. Dose distribution within the anthropomorphic phantom as measured with the EBT2 film and the XR-RV3 film respectively.

\section{ACKNOWLEDGENT}

Authors of the paper would like to acknowledge the assistance provided by Dr. David Lewis regarding information concerning the XRRV3 film and the EBT2 film. This research project was funded by the South African Medical Research Council with funds from National Treasury under its Economic Competitiveness and Support Package. This research and the publication thereof is the result of funding provided by the Medical Research Council of South Africa in terms of the MRC's Flagships Awards Project SAMRC-RFA-UFSP-01-2013/HARD.

\section{Conflict of interest: Declared none.}

\section{REFERENCES}

1. Pai S, Das IJ, Dempsey JF, Lam KL, LoSasso TJ, Olch AJ, et al. (2007) TG-69: Radiographic film for megavoltage beam dosimetry. Med Phys, 34(6):2228-58.

2. Chetty IJ and Charland PM (2002) Investigation of Kodak extended dose range (EDR) film for megavoltage photon beam dosimetry. Phys Med Biol, 47(20):3629.

3. Rink A, Lewis DF, Varma S, Vitkin IA, Jaffray DA (2008)
Temperature and hydration effects on absorbance spectra and radiation sensitivity of a radiochromic medium. Med Phys, 35(10): 4545-55.

4. Odian G (2004) Principles of Polymerization. Wiley, $832 p$.

5. Reinstein LE, Gluckman GR, Meek AG (1998) A rapid colour stabilization technique for radiochromic film dosimetry. Phys Med Biol, 43(10): 2703-8.

6. McCabe BP, Speidel MA, Pike TL, Lysel MSV (2011) Calibration of GafChromic XR-RV3 radiochromic film for skin dose measurement using standardized X-ray spectra and a commercial flatbed scanner. Med Phys, 38 (4):191930.

7. Neocleous A, Yakoumakis E, Gialousis G, Dimitriadis A, Yakoumakis N, Georgiou E (2011) Dosimetry using Gafchromic XR-RV2 radiochromic films in interventional radiology. Radiat Prot Dosimetry, 147(1-2):78-82.

8. Arjomandy B, Tailor R, Anand A, Sahoo N, Gillin M, Prado $K$, et al. (2010) Energy dependence and dose response of Gafchromic EBT2 film over a wide range of photon, electron, and proton beam energies. Med Phys, 37(5):1942-7.

9. Brown TAD, Hogstrom KR, Alvarez D, Matthews KL, Ham K, Dugas JP (2012) Dose-response curve of EBT, EBT2, and EBT3 radiochromic films to synchrotron-produced monochromatic X-ray beams. Med Phys, 39(12): 7412-7.

10. Alnawaf H, Yu PKN, Butson M (2012) Comparison of Epson scanner quality for radiochromic film evaluation. $J$ Appl Clin Med Phys Am Coll Med Phys, 13 (5):3957.

11. Park S, Kang S-K, Cheong K-H, Hwang T, Kim H, Han T, et al. (2012) Variations in dose distribution and optical properties of Gafchromic[sup TM] EBT2 film according to scanning mode. Med Phys, 39(5):2524-35.

12. Mendez I, Hartman V, Hudej R, Strojnik A, Casar B (2013) Gafchromic EBT2 film dosimetry in reflection mode with a novel plan-based calibration method. Med Phys,40(1): 011720.

13. Yarahmadi M, Nedaie HA, Allahverdi M, Asnaashari K, Sauer OA (2013) Small photon field dosimetry using EBT2 Gafchromic film and Monte Carlo simulation. Int. J Radiat Res, 11(4): 215-24.

14. Dreindl R, Georg D, Stock M (2014) Radiochromic film dosimetry: Considerations on precision and accuracy for EBT2 and EBT3 type films. Z Für Med Phys, 24(2):153-63.

15. Girard F, Bouchard H, Lacroix F (2013) Reference dosimetry using radiochromic film. J Appl Clin Med Phys [Internet]. (2012)13(6). Available from: http:// www.jacmp.org/index.php/jacmp/article/view/3994.

16. Lewis D, Micke A, Yu X, Chan MF (2012) An efficient protocol for radiochromic film dosimetry combining calibration and measurement in a single scan. Med Phys, 39(10): 6339-50. 